\title{
Remifentanil or dexmedetomidine for monitored anesthesia care during cataract surgery under topical anesthesia
}

\author{
Ju-hee Park, and Jae-Young Kwon \\ Department of Anesthesia and Pain Medicine, Pusan National University School of Medicine, Busan, Korea
}

Cataract surgery is usually performed under topical anesthesia. The procedure is quick and simple to perform and topical anesthesia avoids the potential hazards of a nerve block. However, topical anesthesia may not provide complete anesthesia and may cause anxiety or uncontrolled and unexpected movement. Monitored anesthesia care (MAC) is intended to provide anxiety relief, patient comfort, and safety during eye surgery. Propofol, benzodiazepines, and opioids have been used for sedation during cataract surgery [1]. However each of these drugs causes respiratory depression, hypoxemia, and apnea. The adverse respiratory complications of these drugs may impede the patient's cooperation during surgery and increase the need for a sedative drug that can be used safely during MAC. Dexmedetomidine is a central $\alpha 2$ agonist that can be titrated to the desired sedation level without significant respiratory depression and has been used as a primary sedative drug for various surgical and diagnostic procedures [2]. Unlike other opioids, remifentanil, which is a $\mu$-opioid receptor agonist, has a short elimination half life of 3-10 min and a short context sensitive half life of 3-5 min. These properties of remifentanil suggest that it may be useful for MAC either combined with propofol or alone [3]. The aim of this study was to compare dexmedetomidine with remifentanil during cataract surgery in patients under topical anesthesia.

The protocol was approved by the Institutional Review Board of our hospital, and all patients provided written informed consent. Eighty American Society of Anesthesiology physical status class $1-3$ patients (age, 60-80 years), who were scheduled for elective cataract surgery under topical anesthesia, were enrolled in this study. Patients were randomized to receive either dexmedetomidine (group D) or remifentanil (group R). Group D patients received an infusion of $0.5 \mu \mathrm{g} / \mathrm{kg}$ dexmedetomidine over 10 minutes followed by a maintenance infusion at a rate of $0.2 \mu \mathrm{g} / \mathrm{kg} / \mathrm{hr}$. Group $\mathrm{R}$ patients received remifentanil by target controlled infusion to reach a $1 \mathrm{ng} / \mathrm{ml}$ target effect site concentration (Cet). Heart rate (HR), systolic blood pressure, diastolic blood pressure, mean arterial pressure (MAP), respiratory rate (RR), oxygen saturation $\left(\mathrm{SpO}_{2}\right)$, bispectral index monitor (BIS) and end tidal (ET) $\mathrm{CO}_{2}$ were recorded before infusion (baseline), after the start of infusion, and every 5 minutes after infusion. The degree of sedation was evaluated using the Ramsay sedation scale by an anesthesiologist who was unaware of the type of sedative used. The satisfaction of the patients and surgeon was assessed immediately after surgery using a 7-point Likert-like verbal rating scale. Recovery time was the interval from stopping the infusion to the time when the patient's Ramsay sedation score was 2. Demographic variables were compared using the t-test. Physiological data were analyzed by repeated-measures analysis of variance. The chi-square test was used to compare the sedation and satisfaction scores.

No differences in baseline characteristics were observed between the groups. Significantly lower MAP, $\mathrm{ETCO}_{2}$, and higher RR were observed over time in group D compared with those in group R. No significant differences in $\mathrm{HR}$ or $\mathrm{SpO}_{2}$ were observed between the groups. BIS scores decreased at 10, 15, and 20 minutes after infusion, but no significant differences in BIS scores were observed between the groups. More patients

Corresponding author: Jae-Young Kwon, M.D., Department of Anesthesia and Pain Medicine, Pusan National University School of Medicine, 179, Gudeok-ro, Seo-gu, Busan 602-739, Korea. Tel: 82-51-240-7398, Fax: 82-51-242-7466, E-mail: jykwon@pusan.ac.kr

(c) This is an open-access article distributed under the terms of the Creative Commons Attribution Non-Commercial License (http:// creativecommons.org/licenses/by-nc/3.0/), which permits unrestricted non-commercial use, distribution, and reproduction in any medium, provided the original work is properly cited. 
Table 1. Patient and Surgeon Satisfaction Scale

\begin{tabular}{|c|c|c|c|c|}
\hline \multirow{2}{*}{$\begin{array}{l}\text { A 7-point Likert-like } \\
\text { verbal rating scale }\end{array}$} & \multicolumn{2}{|c|}{ Surgeon's satisfaction scale* } & \multicolumn{2}{|c|}{ Patient's satisfaction scale } \\
\hline & R group (n) & $\mathrm{D}$ group $(\mathrm{n})$ & $\mathrm{R}$ group (n) & $D$ group $(n)$ \\
\hline 1 & 0 & 0 & 0 & 0 \\
\hline 2 & 0 & 2 & 0 & 0 \\
\hline 3 & 1 & 3 & 1 & 0 \\
\hline 4 & 0 & 6 & 1 & 4 \\
\hline 5 & 2 & 9 & 3 & 3 \\
\hline 6 & 19 & 13 & 13 & 20 \\
\hline 7 & 18 & 7 & 22 & 13 \\
\hline
\end{tabular}

No difference in patient satisfaction scores were observed between the groups. However surgeon satisfaction scores were higher in group $\mathrm{R}$ compared with those in group D. ${ }^{*} \mathrm{P}<0.05$ between the groups, 7-Point Likert-Like Verbal Rating Scale: 1, extremely dissatisfied; 2 , dissatisfied; 3 , somewhat dissatisfied; 4 , undecided; 5 , somewhat satisfied; 6 , satisfied; 7 , extremely satisfied.

in group $\mathrm{D}$ had higher sedation scores at 10 minutes compared with patients in group $\mathrm{R}(\mathrm{P}<0.05)$. No difference in patient satisfaction scores were found between the groups. However, the surgeon's satisfaction scores were higher in group $\mathrm{R}$ compared with those in group $\mathrm{D}(\mathrm{P}<0.05$, Table 1$)$. Recovery times were longer in group $\mathrm{D}(7.0-8.0 \mathrm{~min})$ compared with those in group $\mathrm{R}(1.9-1.7 \mathrm{~min})$.

Ryu et al. [4] found that remifentanil with $1.0 \mu \mathrm{g} / \mathrm{ml}$ propofol at a Cet of $1.0 \mathrm{ng} / \mathrm{ml}$ provides adequate analgesia for patients and excellent operator conditions during cataract surgery. In our study, remifentanil at a Cet of $1.0 \mathrm{ng} / \mathrm{ml}$ alone provided adequate analgesia and conscious sedation. Additional sedative was not administered, because patient cooperation was needed for the operation. Dexmedetomidine also provided adequate analgesia and sedation in this study. Patients sedated with dexmedetomidine are usually fully cooperative, but patients in the dexmedetomidine-treated group in the present study were more sedated than those in group R. As spontaneous ocular movement is common and surgical procedures are frequently hindered at Ramsay scores of 4 or 5 , the surgeon preferred a Ramsay sedation score of 2 or 3 during the operation. Higher surgeon satisfaction was observed in group $\mathrm{R}$ compared with that in group $\mathrm{D}$, which may have been related to patient cooperation during the operation. Recovery times were longer in group $\mathrm{D}$ than those in the other groups. This finding could be attributed to sustained therapeutic plasma concentrations of dexmedetomidine, which has a longer context-sensitive halftime compared with that of remifentanil. The context-sensitive half time of remifentanil is about 3.5 minutes regardless of infusion time. In contrast, the elimination half life of dexmedetomidine is $2-3$ hours, with a context-sensitive halftime ranging from 4 minutes after a 10 minute infusion to 250 minutes after an 8 hour infusion [5].

In conclusion, the surgeon's satisfaction was lower for dexmedetomidine sedation because of poorer cooperation and deep sedation of the patients. Remifentanil may provide better sedation and patient cooperation compared to those of dexmedetomidine during cataract surgery for patients under topical anesthesia.

\section{References}

1. Janzen PR, Christys A, Vucevic M. Patient-controlled sedation using propofol in elderly patients in day-case cataract surgery. Br J Anaesth 1999; 82: 635-6.

2. Alhashemi JA. Dexmedetomidine vs. midazolam for monitored anesthesia care during cataract surgery. Br J Anaesth 2006; 96: 7226

3. Holas A, Krafft P, Marcovic M, Quehenberger F. Remifentanil, propofol or both for conscious sedation during eye surgery under regional anaesthesia. Eur J Anaesthesiol 1999; 16: 741-8.

4. Ryu JH, So YM, Hwang JW, Do SH. Optimal target concentration of remifentanil during cataract surgery with monitored anesthesia care. J Clin Anesth 2010; 22: 533-7.

5. Venn RM, Karol MD, Grounds RM. Pharmacokinetics of dexmedetomidine infusions for sedation of postoperative patients requiring intensive care. Br J Anaesth 2002; 88: 669-75. 